\title{
Nonlinear Dynamic Characteristics of Giant Magnetostrictive Sensor
}

\author{
Zhiwen $\mathrm{Zhu}^{1,2}$, Wendi Zhang ${ }^{1}$ and Jia $\mathrm{Xu}^{1, *}$ \\ 'Department of Mechanics, Tianjin University, Tianjin 300072, China \\ ${ }^{2}$ Tianjin Key Laboratory of Nonlinear Dynamics and Chaos Control, Tianjin 300072, China
}

(Received December 24, 2013; accepted March 6, 2014)

Key words: giant magnetostrictive sensor, hysteretic nonlinearity, partial least-squares regression

In this study, a type of hysteretic nonlinear model of giant magnetostrictive materials (GMMs) based on the inverse magnetostrictive effect was developed, and the nonlinear dynamic characteristics of a giant magnetostrictive sensor were studied. A Van der Pol nonlinear item was introduced to describe the hysteretic phenomena of GMMs, and the coupling relationship between strain and frequency was determined. The results of a forecast test show that the GMM model can describe well the inverse magnetostrictive effect in different frequencies. On the basis of the GMM model, the magnetomechanical coupled model of a giant magnetostrictive sensor was developed, and the relationship between output voltage and input excitation force was determined. The nonlinear dynamic characteristics of the giant magnetostrictive sensor were discussed, and the phenomena of accuracy aggravation in high frequency and delay of a giant magnetostrictive sensor were explained. The experimental results show that the model can describe the actual response of the giant magnetostrictive sensor. The new model of the giant magnetostrictive sensor has a simple form and is easy to analyze in theory, which is helpful for application in measure and control fields.

\section{Introduction}

A giant magnetostrictive material (GMM) converts mechanical energy into magnetic energy, which is known as the inverse magnetostrictive effect or the Villari effect. On the basis of this effect, giant magnetostrictive sensors used in micro-electromechanical systems (MEMS) can be designed. Giant magnetostrictive sensors have many advantages, such as small size, rapid response, long service life, and wide range of measurement, and are widely applied in vibration measurement fields. ${ }^{(1)}$

Many scholars have studied giant magnetostrictive sensors. Jia et al. designed a novel force sensor based on a giant magnetostrictive material.(2) Yan et al. developed the dynamic model of giant magnetostrictive acceleration sensors including Eddy-Current effects. ${ }^{(3)}$ Huang et al. developed novel sensors based on magnetostrictive/piezoelectric laminations. ${ }^{(4)}$ Pacheco and Bruno studied the effect of shape anisotropy in giant

${ }^{*}$ Corresponding author: e-mail: xujia_ld@163.com 
magnetostrictive fiber Bragg grating sensors. ${ }^{(5)}$ Although many advances have been obtained, the modeling problem of the giant magnetostrictive sensor limits its application in industry. ${ }^{(6)}$ To optimize giant magnetostrictive sensors effectively, it is necessary to build its model with high accuracy.

Most of the physical models of GMM were shown as equations with subsection function or double integral function, which were difficult to analyze in theory owing to the hysteretic characteristics of GMM..$^{(7-9)}$ Usually, research results could only be obtained by numerical or experimental methods. ${ }^{(10-12)}$ In this study, a Van der Pol nonlinear item was introduced to interpret the hysteretic phenomenona of GMM, and the dynamic characteristics of the giant magnetostrictive sensor were studied.

\section{Hysteretic Nonlinear Model of Giant Magnetostrictive Material}

The strain-magnetic field intensity (MFI) curve of GMM is shown in Fig. 1. In this study, a Van der Pol hysteretic model was introduced to describe the hysteretic nonlinear characteristics of GMM based on the inverse magnetostrictive effect.

The strain-MFI curve of GMM can be shown as follows when it is supposed to be symmetrical about the point $\mathrm{G}\left(\varepsilon_{0}, H_{0}\right)$.

$$
H=\varphi \varepsilon+\chi \varepsilon^{2}+\alpha \varepsilon^{3}+\beta\left(\rho \varepsilon-\varepsilon^{2}\right) \dot{\varepsilon}
$$

From Fig. 1, we can see that frequency can also affect the magnetoelasticity of GMM, which means that there are items coupling the strain and frequency. Supposing that all the coupling items will appear in the model, the basic model of $H$ can be shown as follows.

$$
\begin{aligned}
H= & c_{1} \varepsilon+c_{2} \varepsilon^{2}+c_{3} \varepsilon^{3}+c_{4} \varepsilon \dot{\varepsilon}+c_{5} \varepsilon^{2} \dot{\varepsilon}+c_{6} \varepsilon f+c_{7} \varepsilon^{2} f+c_{8} \varepsilon^{3} f \\
& +c_{9} \varepsilon \dot{\varepsilon} f+c_{10} \varepsilon^{2} \dot{\varepsilon} f+c_{11} \varepsilon f^{2}+c_{12} \varepsilon^{2} f^{2}+c_{13} \varepsilon^{3} f^{2}+c_{14} \varepsilon \dot{\varepsilon} f^{2} \\
& +c_{15} \varepsilon^{2} \dot{\varepsilon} f^{2}+c_{16} \varepsilon f^{3}+c_{17} \varepsilon^{2} f^{3}+c_{18} \varepsilon^{3} f^{3}+c_{19} \varepsilon \dot{\varepsilon} f^{3}+\varepsilon_{20} \varepsilon^{2} \dot{\varepsilon} f^{3}
\end{aligned}
$$

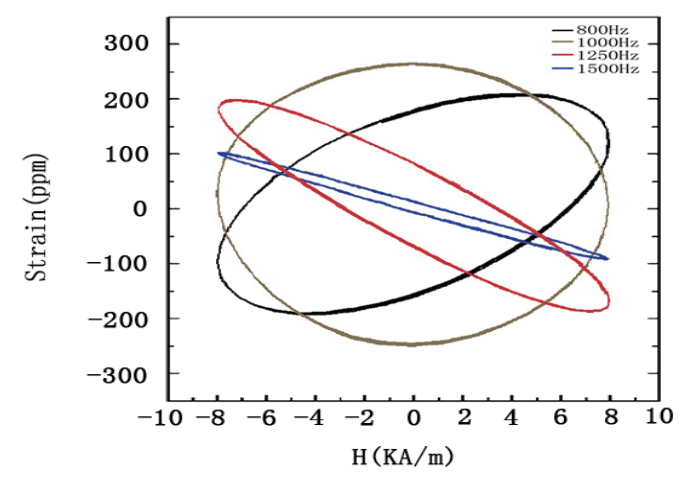

Fig. 1. (Color online) Strain-MFI curves of GMM at different frequencies. 
In this study, a partial least-squares regression method was applied in finding the concise coupling relationship between strain and frequency. Reading experimental data from Fig. 1 and regarding the items in eq. (2) as independent variables, we obtained the analysis result of the principal component, which is shown in Fig. 2.

According to variable importance, the variables that are the most significant $(V I P>0.8)$ in the basic model were chosen as follows: $\varepsilon, \varepsilon^{2}, \varepsilon^{3}, \varepsilon \cdot \dot{\varepsilon}, \varepsilon^{2} \cdot \dot{\varepsilon}$, and $\varepsilon^{3} \cdot f$. Thus, the final relationship among strain, MFI and frequency can be shown as follows.

$$
H=a_{1} \varepsilon+a_{2} \varepsilon^{2}+a_{3} \varepsilon^{3}+a_{4} \varepsilon \dot{\varepsilon}+a_{5} \varepsilon^{2} \dot{\varepsilon}+a_{6} \varepsilon^{3} f
$$

The result of the forecast test using eq. (3) is shown in Fig. 3, where the red line represents real data and the black line indicates forecast value. Obviously, eq. (3) can describe the real curve well.

\section{Dynamic Characteristics of Giant Magnetostrictive Sensor}

A typical structure of the giant magnetostrictive sensor is shown in Fig. 4, and the mechanical model of the giant magnetostrictive sensor is shown in Fig. 5, where $m_{\mathrm{r}}$ is

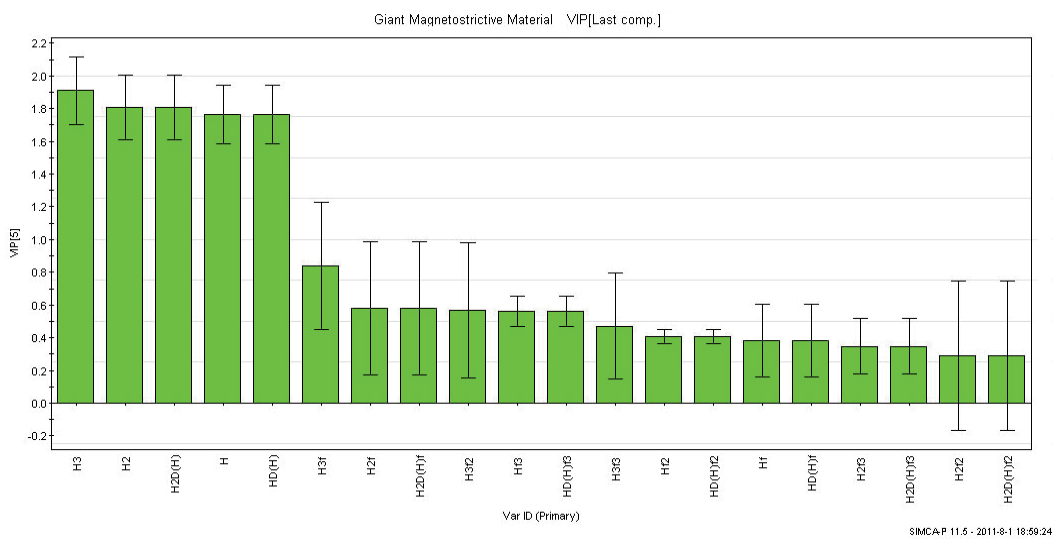

Fig. 2. (Color online) Analysis result of principal component.

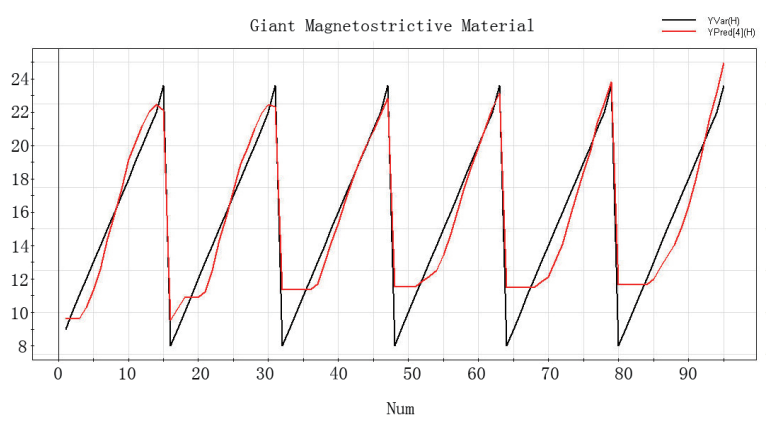

Fig. 3. (Color online) Result of forecast test. 

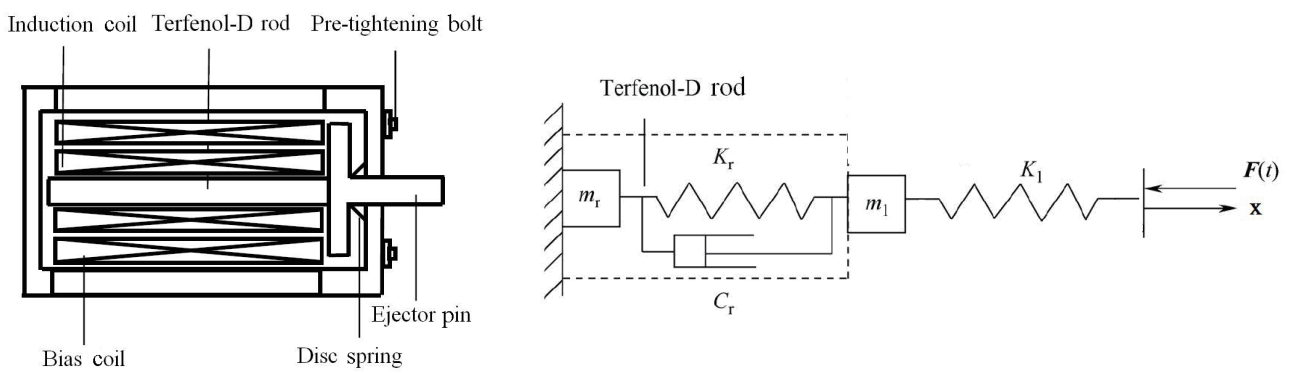

Fig. 4 (left). Structure of giant magnetostrictive sensor.

Fig. 5 (right). Mechanical model of giant magnetostrictive sensor with load.

the mass of the Terfenol-D rod, $m_{1}$ is the equivalent mass of the load, $C_{\mathrm{r}}$ is the structural damping of the sensor, $C_{1}$ is the equivalent damping of the load, $k_{\mathrm{r}}$ is the stiffness of the Terfenol-D rod, $k_{1}$ is the equivalent stiffness of the load, and $F(t)$ is the excitation force.

The dynamic model of the giant magnetostrictive sensor can be shown as

$$
\left[\begin{array}{cc}
m_{\mathrm{r}} & 0 \\
0 & m_{\mathrm{r}}
\end{array}\right]\left(\begin{array}{c}
\ddot{x}_{\mathrm{r}} \\
\ddot{x}_{1}
\end{array}\right)+\left[\begin{array}{cc}
c_{\mathrm{r}} & 0 \\
0 & 0
\end{array}\right]\left(\begin{array}{c}
\dot{x}_{\mathrm{r}} \\
\dot{x}_{1}
\end{array}\right)+\left[\begin{array}{cc}
k_{1}+k_{\mathrm{r}} & -k_{1} \\
-k_{1} & k_{1}
\end{array}\right]\left(\begin{array}{c}
x_{\mathrm{r}} \\
x_{1}
\end{array}\right)=\left(\begin{array}{c}
0 \\
F(t)
\end{array}\right)
$$

where $x_{\mathrm{r}}$ is the giant magnetostrictive sensor and $x_{1}$ is the load displacement.

If $F=F(t)=\bar{F} \cos \omega$, we obtain

$$
x_{\mathrm{r}}=l \varepsilon=\frac{\sqrt{\left[k_{1}\left(m_{1}+m_{\mathrm{r}}\right)-\omega^{2} m_{1} m_{\mathrm{r}}\right]^{2}+\omega^{2} c_{\mathrm{r}}^{2}\left(m_{1}+m_{\mathrm{r}}\right)^{2}}}{\sqrt{\bar{a}^{2}+\bar{b}^{2}}} \overline{c o s} \omega t=n_{1} \bar{F} \cos \omega t
$$

where

$$
\begin{gathered}
n_{1}=\frac{\sqrt{\left[k_{1}\left(m_{1}+m_{\mathrm{r}}\right)-\omega^{2} m_{1} m_{\mathrm{r}}\right]^{2}+\omega^{2} c_{\mathrm{r}}^{2}\left(m_{1}+m_{\mathrm{r}}\right)^{2}}}{\sqrt{\bar{a}^{2}+\bar{b}^{2}}} \\
\bar{a}=\omega^{4} m_{1} m_{\mathrm{r}}-\omega^{2}\left(2 k_{1} m_{\mathrm{r}}+k_{\mathrm{r}} m_{1}+k_{1} m_{1}\right)+2 k_{1}\left(k_{\mathrm{r}}+k_{1}\right) \\
\bar{b}=\omega^{2} c_{\mathrm{r}}\left(m_{1}+m_{\mathrm{r}}\right)+\omega c_{\mathrm{r}}\left(3 k_{1}-k_{\mathrm{r}}\right)
\end{gathered}
$$

According to eq. (5), the output voltage $U$ of the giant magnetostrictive sensor can be shown as

$$
U=\delta H=\delta\left(a_{1} \varepsilon+a_{2} \varepsilon^{2}+a_{3} \varepsilon^{3}+a_{4} \varepsilon \dot{\varepsilon}+a_{5} \varepsilon^{2} \dot{\varepsilon}+a_{6} \varepsilon^{3} f\right)=U_{0}+U_{\mathrm{L}}+U_{\mathrm{NL}},
$$


where $\delta$ is the magnification coefficient.

$$
\begin{gathered}
U_{0}=\frac{\delta a_{2} n_{1}^{2}}{2 l^{2}} \bar{F}^{2} \\
U_{\mathrm{L}}=\delta \frac{a_{1} n_{1}}{l} \bar{F} \cos \omega t \\
U_{\mathrm{NL}}=\frac{3\left(a_{3}+a_{6} f\right) n_{1}^{3}}{4 l^{3}} \bar{F}^{3} \cos \omega t+\frac{a_{2} n_{1}^{2}}{2 l^{2}} \bar{F}^{2} \cos 2 \omega t+\frac{\left(a_{3}+a_{6} f\right) n_{1}^{3}}{4 l^{3}} \bar{F}^{3} \cos 3 \omega t \\
+\frac{a_{5} n_{1}^{3} \omega}{4 l^{3}} \bar{F}^{3} \sin \omega t-\frac{a_{4} n_{1}^{2} \omega}{2 l^{2}} \bar{F}^{2} \sin 2 \omega t+\frac{a_{5} n_{1}^{3} \omega}{4 l^{3}} \bar{F}^{3} \sin 3 \omega t
\end{gathered}
$$

Now, we can discuss eq. (9).

1) There are three types of frequency component: $\omega, 2 \omega$, and $3 \omega$ in voltage $U$.

2) Owing to the existence of items $\sin \omega t$ and $\cos \omega t$ in $U_{\mathrm{NL}}$, the phase of the voltage waveform is different from that of the excitation force.

3) $U_{0}=\frac{\delta a_{2} n_{1}^{2}}{2 l^{2}} \bar{F}^{2}$ is constant when $\bar{F}$ is determined, and can be adjusted to zero.

4) $U_{L}=\delta \frac{a_{1} n_{1}}{l} \bar{F}$ is linear with $F$, so $U=U_{\mathrm{L}}$ is ideal.

5) $U_{\mathrm{NL}}$ is nonlinear. It is the error between the actual output voltage and the linear scale of the giant magnetostrictive sensor, which is inevitable but can be reduced.

6) From eq. (12), we can see that $U_{\mathrm{NL}}$ is relative to $\omega$. For vibration at low frequency, $\omega$ is small, so $U_{\mathrm{NL}}$ is small. It means that the giant magnetostrictive sensor is approximately linear in measuring vibration in low frequency.

7) $U_{\mathrm{NL}}$ increases with $\omega$. For vibration at high frequency, $U_{\mathrm{NL}}$ is remarkable. It means that the accuracy of the giant magnetostrictive sensor will be exasperated at high frequency, which has been proved by many researchers. ${ }^{(14-16)}$ To reduce the accuracy aggravation, the length of the GMM rod should be increased.

The experimental results of the giant magnetostrictive sensor system are shown in Fig. 6 . We can see that eq. (9) can describe the experiment results. The waveform of the output voltage is not smooth owing to the existence of multiple-frequency components.

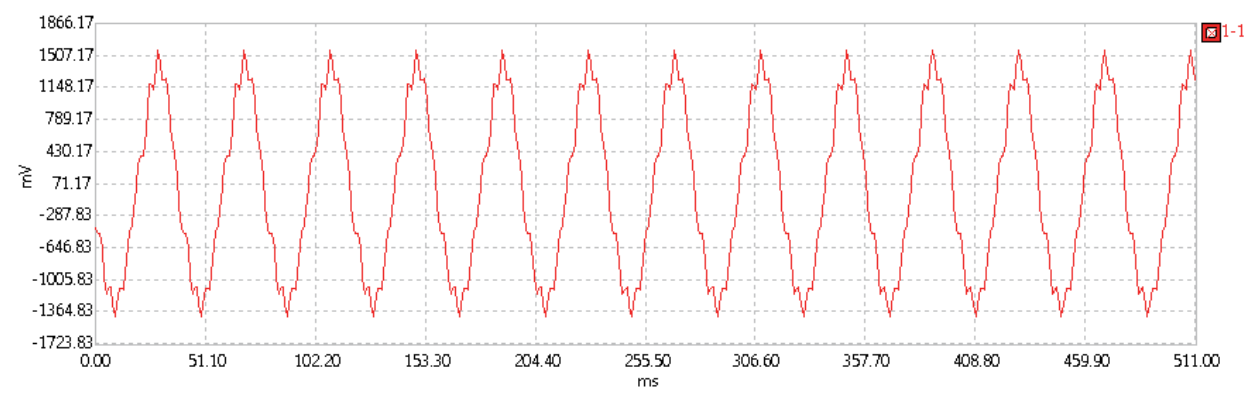

Fig. 6. (Color online) Output voltage curve of giant magnetostrictive sensor. 


\section{Conclusions}

In this study, a type of hysteretic nonlinear model of GMMs based on the inverse magnetostrictive effect was developed, and the nonlinear dynamic characteristics of a giant magnetostrictive sensor were studied. A Van der Pol nonlinear item was introduced to describe the hysteretic phenomena of GMM, and the coupling relationship between strain and frequency was determined. The results of the forecast test show that the GMM model can describe well the inverse magnetostrictive effect at different frequencies. On the basis of the GMM model, the magneto-mechanical coupled model of a giant magnetostrictive sensor was developed, and the relationship between output voltage and input excitation force was determined. The nonlinear dynamic characteristics of the giant magnetostrictive sensor were discussed, and the phenomena of accuracy aggravation in high frequency and delay of giant magnetostrictive sensor were explained. The experimental results show that the model can describe the actual response of the giant magnetostrictive sensor. The new model of the giant magnetostrictive sensor has a simple form and is easy to analyze in theory, which is helpful for application in measure and control fields.

\section{Acknowledgements}

The authors gratefully acknowledge the support of the Natural Science Foundation of China (NSFC) through grant nos. 11272229 and 11302144, the Ph.D. Programs Foundation of the Ministry of Education of China through grant no. 20120032120006, and the Tianjin Research Program of Application Foundation and Advanced Technology through grant no. 13JCYBJC17900.

\section{References}

1 U. Toshiyuki, J. H. Qiu and T. Junji: IEEE Trans. Magn. 40 (2004) 1601.

2 Z. Y. Jia, H. F. Liu and F. J. Wang: J. Alloy. Compd. 509 (2011) 1760.

3 R. G. Yan, Q. X. Yang and W. R. Yang: IEEE T. Appl. Supercon. 20 (2010) 1874.

4 J. K. Huang, D. Bono and R. C. O'Handley: Sens. Lett. 5 (2007) 105.

5 C. J. Pacheco and A. C. Bruno: Meas. Sci. Technol. 21 (2010) 065205.

6 Y. Y. Kim, S. Y. Cho and H. C. Lee: J. Sound. Vib. 268 (2003) 799.

7 D. C. Jiles and D. L. Atherton: J. Magn. Magn. Mater. 61 (1986) 48.

8 G. P. Carman and M. Mitrovic: J. Intel. Mat. Syst. Str. 6 (1995) 673.

9 F. T. Calkins, R. C. Smith and A. B. Flatau: IEEE Trans. Magn. 36 (2000) 429.

10 D. H. Sul, Y. W. Park and H. J. Park: IEEE Trans. Magn. 43 (2007) 2591.

11 M. E. H. Benbouzid, R. Gilbert and M. Gerard: IEEE Trans. Magn. 31 (1995) 1821.

12 P. G. Evans and M. J. Dapino: J. Appl. Phys. 107 (2010) 063906. 